\title{
Thick Strip Method for Efficient Large-Eddy Simulations of Flexible Wings in Stall
}

\author{
Mohsen Lahooti* and Rafael Palacios ${ }^{\dagger}$ and Spencer J. Sherwin ${ }^{\ddagger}$ \\ Imperial College, London, SW7 2AZ, United Kingdom
}

\begin{abstract}
Efficient computational method is presented based on the "thick" strip method for large eddy simulation of flexible wings in stall. Fluid domain is break down into series of smaller $3 D$ strips where can be efficiently solved with LES method for fluid flow. Force and moments are obtained from each strips and used to evolved the nonlinear dynamics of the wings. Preliminary result presented for high-altitude long-endurance wing which clearly shows the capability and robustness of presented approach.
\end{abstract}

\section{Introduction}

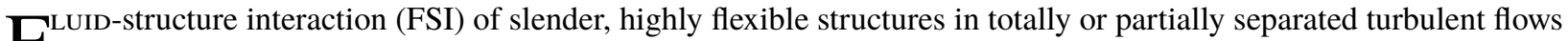

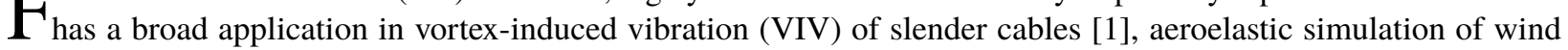
turbine blades [2] and high-altitude long-endurance (HALE) aircraft aeroelastic simulations [3] to name a few. Such FSI simulations are computationally challenging in terms of accuracy, robustness of numerical scheme and computational costs. Currently, several approaches are available for such FSI simulations including semi-empirical models [4-6], which are built upon experimental results and hence they are unable to capture the intrinsic physical mechanisms involved and may lack a predictable capability to analyse new configurations. Other commonly used approaches such as Blade Element Momentum (BEM) theory for wind turbine applications [7, 8] or unsteady thin-airfoil theory with a dynamic stall model [9, 10] are based on simplified two-dimensional theories and hence unable to capture the finite-length and flow unsteadiness effects. Vortex methods [11] and Unsteady Vortex Lattice Method [2] 12] are able to capture the three-dimensional effects, however, being based on potential theory they are suitable for preliminary studies. More sophisticated computational approaches such as RANS or LES method for solution of Navier-Stokes equations to capture the flow dynamics and predict aerodynamic forces on the structure are computationally expensive where the former one is unable to provide a resolved information of turbulent flow. LES method is extremely resourceful and computationally expensive to well resolve the flow and maintain the required resolution along the spanwise direction of the structure. LES require significant grids for full $3 D$ simulation due to the large spanwise length of slender structure which makes them impractical for FSI simulation of real-size applications with high-Reynolds-number flows.

A computationally efficient approach to address this challenge is to use the thick strip method proposed by Bao et al.[1] for VIV of slender cylinders. The thick strip method is basically generalisation of strip-based theory methods. where the fluid domain is divided into a series of domains along the structure's spanwise length. Each thick strip is a three-dimensional domain perpendicular to the structure's local axis with a finite size thickness. The finite size of each strips enables capturing local spanwise velocity correlations and reflecting the wake turbulence on structural dynamics. On the other hand, breaking down the full $3 D$ problems into series of locally $3 D$ domains, provides a computationally efficient approach for simulation of such problems. In the thick strip method of Bao et al. [1], fluid flow is solved using DNS method and structural dynamics is modelled using Discrete Fourier Transform (DFT) of linearised Euler beam. Motivated by computational efficiency of thick strip method, in the present work the method is extended to handle highly deformable geometrically nonlinear slender structures for simulation of HALE wings in stall. The flow solver is implemented in high-order spectral/hp element framework-Nektar++ [13] and coupled with the multi-body geometrically nonlinear structural solver SHARPy [14, 15].

The rest of the paper is organised as follows: section $\Pi$ summarize the thick strip method and provides a brief description of governing equations and solution procedure for fluid, solid and FSI solver. IIIIdescribes the test case and provides preliminary results for the HALE wing deformation along with a detailed discussion on the three-dimensional effects on the results. Finally, section [V] outlines the future work for the full paper.

\footnotetext{
*Research Associate, Department of Aeronautics. m.lahooti@imperial.ac.uk

†Professor, Department of Aeronautics, AIAA associate member, r.palacios@imperial.ac.uk.

†Professor, Department of Aeronautics. s.sherwin@imperial.ac.uk
} 


\section{Thick strips FSI method}

The $3 D$ fluid domain is divided into a series of independent domains along the structure's spanwise length. Each strip is a three-dimensional domain consisting of two-dimensional domains perpendicular to the structure's local axis with a finite size thickness of $L_{z}$ as shown schematically in Fig. 1. The strips are distanced apart from each other with the gap size of $L_{g}$ which is related to the total structural length through Eq. 1 as

$$
L_{s}=L_{z}+(N-1) \times\left(L_{z}+L_{g}\right)
$$

where $L_{s}$ is the total length of slender structure, $N$ is the number of strips and, $L_{z}$ and $L_{g}$ are strips thickness and gap size between strips respectively. Theses separated flow-domains are implicitly connected through the structural dynamics and hence enable a three-dimensional FSI simulation of slender structures in turbulent flows. A distinct advantage of such an approach is that only a fraction of a full three-dimensional fluid domain is modelled and it is further broken down into smaller domains, which can be simulated efficiently. Furthermore, considering a finite size thickness $\left(L_{z}\right)$ for each domain enables the spanwise velocity correlations to be constructed locally. Hence, the effect of wake turbulence would be adequately taken into account while avoiding a costly and time-consuming full three-dimensional simulation.

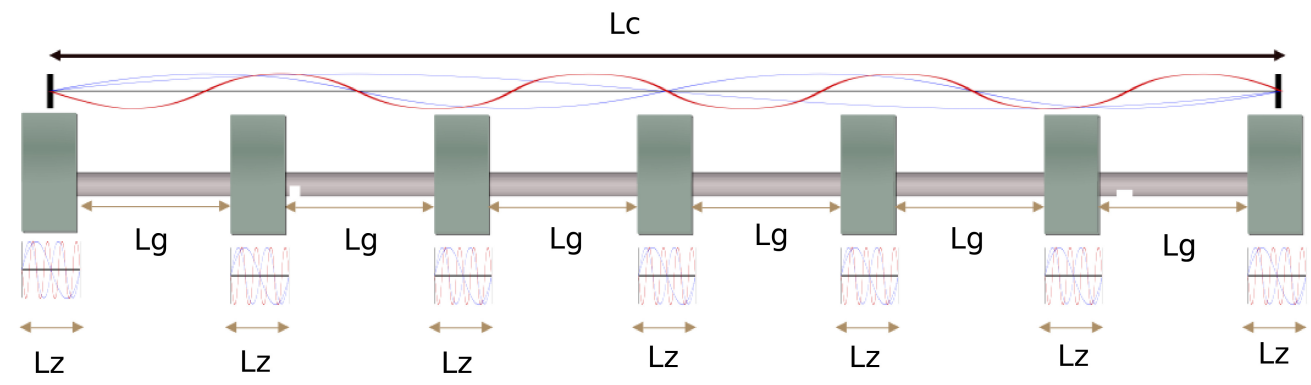

Fig. 1 Schematic of thick strip method for a three-dimensional simulation of Fluid-Structure Interaction of turbulent flow with long slender flexible structure

\section{A. Fluid solver}

The fluid flow is governed by the Navier-Stokes equations in each fluid strip domain $\bar{\Omega}_{n}(t)$ are as

$$
\begin{aligned}
\frac{\partial \overline{\mathbf{u}}_{n}}{\partial t}+\overline{\mathbf{u}}_{n} \cdot \bar{\nabla} \overline{\mathbf{u}}_{n} & =\frac{-1}{\rho} \bar{\nabla} p_{n}+v \bar{\nabla} \overline{\mathbf{u}}_{n} \quad \text { on } \quad \bar{\Omega}_{n}, \quad n=0 \cdots N-1 \\
\bar{\nabla} \cdot \overline{\mathbf{u}}_{n} & =0
\end{aligned}
$$

where $\overline{\mathbf{u}}$ is the velocity vector and the over bar refers to the formulation in inertial coordinate frame $\overline{\mathbf{X}}=(\bar{x}, \bar{y}, \bar{z})$ with $\bar{z}$ defined along the spanwise coordinates, $p$ is the pressure, $\rho$ and $v$ are fluid density and kinematic viscosity respectively and subscript $n$ represents the $n^{\text {th }}$ fluid strip domain. To solve the coupled FSI system and in order to avoid dynamic re-meshing due to the structural movement and deformation, the Navier-Stokes equations [Eq. [3] ] are transformed into non-inertial body-fitted coordinates [16, 17] as

$$
\bar{\Omega}_{n}(t) \longmapsto \Omega_{n}: x=\bar{x}-\xi_{n}(\bar{z}, t), \quad y=\bar{y}-\eta_{n}(\bar{z}, t), \quad z=\bar{z}
$$

where $\xi_{n}(\bar{z}, t)$ and $\eta_{n}(\bar{z}, t)$ are the coordinates of moving structure in streamwise and cross flow directions respectively. Using the linear mapping in Eq. (4), the flow variables in new coordinate system becomes

$$
u_{n}=\bar{u}_{n}-\frac{\partial \xi_{n}(\bar{z}, t)}{\partial t}, \quad v_{n}=\bar{v}_{n}-\frac{\partial \eta_{n}(\bar{z}, t)}{\partial t}, \quad w_{n}=\bar{w}, \quad p_{n}=\bar{p}
$$


Therefore using the new body-fitted coordinate $\mathbf{X}=(x, y, z)$ (Eq. 4) with the transformed flow variables $(\mathbf{u}, p)=$ $(u, v, w, p)$, the Navier-Stokes equations in the body-fitted coordinates becomes

$$
\begin{aligned}
\frac{\partial \mathbf{u}_{n}}{\partial t}+\mathbf{u}_{n} \cdot \nabla \mathbf{u}_{n} & =\frac{-1}{\rho} \nabla p_{n}+v \nabla^{2} \mathbf{u}_{n}+\mathbf{f}_{n} \quad \text { on } \quad \Omega_{n}, \quad n=0 \cdots N-1 \\
\nabla \cdot \mathbf{u}_{n} & =0
\end{aligned}
$$

where $\mathbf{f}_{n}$ are the source term in each strip arise from the mapping transformations Eq. (4) and Eq. (5), which take into account the non-inertial effects of the new coordinate system, and are defined as

$$
f_{n x}=-\frac{\partial^{2} \xi\left(\bar{z}_{n}, t\right)}{\partial t^{2}} \quad f_{n y}=-\frac{\partial^{2} \eta\left(\bar{z}_{n}, t\right)}{\partial t^{2}} \quad f_{n z}=0
$$

The solution strategy for the thick strip model is to use the Fourier expansion for discretization of equations in $z$ direction and spectral/hp element method in $(x, y)$ planes. Using this approach implies the assumption that the flow variables are homogenous in spanwise direction with a periodic length of $L_{z}$ equal to the length of thick strips. Based on this assumption a Fourier expansion in $z$ direction can be introduced for flow variables as

$$
\begin{aligned}
& \mathbf{u}_{n}(\mathbf{x}, t)=\sum_{m=0}^{M-1} \hat{\mathbf{u}}_{\mathbf{n m}}(x, y, t) \mathrm{e}^{i \beta m z} \\
& p_{n}(\mathbf{x}, t)=\sum_{m=0}^{M-1} \hat{p}_{n m}(x, y, t) \mathrm{e}^{i \beta m z}
\end{aligned}
$$

where $\beta=2 \pi / L_{z}, m$ is the Fourier mode index and $M$ is the total number of Fourier modes.

Applying the Fourier expansions for velocity and pressure (Eqs. (9) and (10) ) to Eqs. (6) and (7) results in a set of 2D uncoupled equations for each mode as

$$
\begin{aligned}
\frac{\partial \hat{\mathbf{u}}_{n m}}{\partial t}+{\widehat{\mathbf{N}\left(\mathbf{u}_{n}\right)_{m}}} & =\frac{-1}{\rho} \tilde{\nabla} \hat{p}_{n m}+v \tilde{\nabla}^{2} \hat{\mathbf{u}}_{n m}+\hat{\mathbf{f}}_{n m} \quad \text { on } \quad \Omega_{n}, \quad n=0 \cdots N-1 \\
\tilde{\nabla} \cdot \hat{\mathbf{u}}_{n m} & =0
\end{aligned}
$$

where $\widehat{\mathbf{N}\left(\mathbf{u}_{n}\right)_{m}}$ and $\hat{\mathbf{f}}_{n m}$ represents the Fourier modes of convective terms and body forces respectively and the gradient and Laplacian operators are defined as

$$
\tilde{\nabla}=\left(\frac{\partial}{\partial x}, \frac{\partial}{\partial y}, i \beta m\right), \quad \tilde{\nabla}^{2}=\frac{\partial^{2}}{\partial x^{2}}+\frac{\partial^{2}}{\partial y^{2}}-\beta^{2} m^{2}
$$

Equation (11) is then discretized on each Fourier plane using the spectral/hp method. Finally, the continuity constraint in incompressible Navier-Stokes equation is enforced via the stiffly stable high-order splitting velocity correction scheme proposed by Karniadakis et al. [18]. For the detail description of the thick strip method and spectral/hp element method refer to [1, 19].

\section{B. Structural dynamics}

The slender structure dynamics can be modelled as first approximation using the Geometrically-Exact Composite Beam (GECB)[15, 20, 21]. The structural dynamics is described using two different frame of references (FR): $A$, and $B$ where $A$ is a body-fixed frame attached to the structure and $B$ is the local coordinate system. The local orientation of each beam cross section is determined via transformation between $B$ and $A$ suing the Cartesian Rotation Vector (CRV): $\boldsymbol{\Psi}(s, t)$. The CRV is based on Euler's rotation theorem which states that any series of rotations of a rigid body around a fixed point can be reduced to a single rotation, of magnitude $\phi$ around a fixed axis $\mathbf{n}$ resulting in $\boldsymbol{\Psi}=\phi \mathbf{n}$ and hence $\mathbf{C}^{B A}(s, t)=\mathbf{C}(\boldsymbol{\Psi})$ can be computed as [20] 


$$
\mathbf{C}(\boldsymbol{\Psi})=\mathbf{I}+\frac{\sin (\phi)}{\phi} \tilde{\boldsymbol{\Psi}}+\frac{1-\cos (\phi)}{\phi^{2}} \tilde{\mathbf{\Psi}}^{2}
$$

where $\tilde{\boldsymbol{\Psi}}$ is the skew-symmetric matrix of rotation vector $\boldsymbol{\Psi}$. Defining the nodal positions in body fixed frame $A$ as $\mathbf{R}_{A}(s, t)$, strain and curvature vectors can be computed as [20]

$$
\begin{aligned}
& \boldsymbol{\gamma}(s, t)=\mathbf{C}(\boldsymbol{\Psi})(s, t) \mathbf{R}_{\mathbf{A}}^{\prime}(s, t)-\mathbf{C}(\boldsymbol{\Psi})(s, 0) \mathbf{R}_{\mathbf{A}}^{\prime}(s, 0) \\
& \boldsymbol{\kappa}(s, t)=\mathbf{T}(\boldsymbol{\Psi})(s, t) \boldsymbol{\Psi}^{\prime}(s, t)-\mathbf{T}(\boldsymbol{\Psi})(s, 0) \boldsymbol{\Psi}^{\prime}(s, 0)
\end{aligned}
$$

where $\bullet$ ' represents the derivative with respect to arch length and rotational operator $\mathbf{T}$ is defined as[20]

$$
\mathbf{T}(\boldsymbol{\Psi})=\mathbf{I}+\frac{\cos (\phi)-1}{\phi^{2}} \tilde{\mathbf{\Psi}}+\frac{1-\frac{\sin (\phi)}{\phi}}{\phi^{2}} \tilde{\mathbf{\Psi}} \tilde{\Psi}
$$

Finally using Hamiltonian principle and Finite element discretization, the final discrete form of equations is written as[20]

$$
\mathcal{M}_{\eta}(\boldsymbol{\eta}) \ddot{\boldsymbol{\eta}}+\mathbf{Q}_{g y r}(\boldsymbol{\eta})+\mathbf{Q}_{\text {stif }}(\boldsymbol{\eta})=\mathbf{Q}_{\text {ext }}(\boldsymbol{\eta})
$$

where $\boldsymbol{\eta}$ is the the state variable containing all displacements and rotations vector of all nodal displacements, and subscripts gyr, stif and ext refers to gyroscopic, stiffness and external forces respectively. External forces on the body includes both aerodynamic forces calculated from the flow solver and body forces such gravity. Equation (18) is discretized using 3-nodes elements and integrated through time using explicit Newmark- $\beta$ method. For the complete details of formulation and solution procedure refer to [15, 20, 21]

\section{FSI coupling}

For the FSI coupling the aerodynamic forces and moments from fluid flow needs to be calculated. Recalling from sec. II.A that the Navier-Stokes equations are solved on the non-inertial body-fitted coordinate $\mathbf{X}$ [Eq. 4], the flow variables transformed back into the inertial coordinate $\overline{\mathbf{X}}$. Therefore, the aerodynamic forces and moments on each fluid strip $n$ are calculated and averaged over the spanwise coordinates of the strip as

$$
\begin{aligned}
\mathbf{F}_{n}(t) & =\int_{L_{z}^{n}} \oint_{\partial \Omega_{n}}\left(-\bar{p} \overline{\mathbf{n}}+v \nabla \overline{\mathbf{u}}_{n} \cdot \overline{\mathbf{n}}\right) d \Gamma d \bar{z} \quad \bar{z} \in\left[n\left(L_{z}+L_{g}\right), L_{z}+n\left(L_{z}+L_{g}\right)\right] \\
\mathbf{M}_{n}(t) & =\int_{L_{z}^{n}} \oint_{\partial \Omega_{n}}\left(-\bar{p} \overline{\mathbf{n}}+v \nabla \overline{\mathbf{u}}_{n} \cdot \overline{\mathbf{n}}\right) \times r d \Gamma d \bar{z}
\end{aligned}
$$

where $\partial \Omega_{n}$ is the structure boundary with unit normal vector $\mathbf{n}$ and $L_{z}^{n}$ is the length of $n^{\text {th }}$ strip defined as $L_{z}^{n}=$ $\left\{\bar{z} \mid n\left(L_{z}+L_{g}\right) \leq \bar{z} \leq L_{z}+n\left(L_{z}+L_{g}\right)\right\}$. It is worth mentioning that the location and number of fluid strips are not necessarily equal to the location and number of vertices used for discretization of structural equation. Hence, after fluid forces and moments are calculated, their values are interpolated to the structural nodes using the third order spline interpolations

Having the aerodynamic forces and moments on the structure, the FSI algorithm can be summarised as

I. Start of the solution:

a Calculate initial static deformation state of the structure

b Update new geometric coordinates in fluid solver

c Initialise Flow Solver

II. Starting of each time loop:

a. Calculate the body forces due to the structural motion using Eq. (8)

b. Solve fluid flow for one time step via integrating Navier-Stokes equations [Eqs.(6) and (7)]

c. Calculate aerodynamic forces and moments on the structure using Eq. (19)

d. Interpolate aerodynamic forces and motion to the location of structural elements and vertices

e. Update structure state variables by solving dynamic equation [Eq. (18] ] 
f. Repeat steps a.- e. until final time reached

To conclude the FSI algorithm, it should be emphasized that although fluid strips are independent fluid domains, they are implicitly connected via the structural motion. In other words, having the aerodynamic forces and moments from various fluid strips, we are able to compute structural motion, velocity and acceleration which in turn shows their effect via the source term (Eq. 8) in Navier-Stokes equation, providing an implicit means to interconnect the separated fluid domains and account for their effects on each other.

\section{Numerical results}

For the numerical test of the proposed method, nonlinear aeroelasticity of a representative HALE wing is considered with a particular interest in prediction of static stall. The HALE wing considered is firstly introduced by Patil et al.[22] and further analysed by Smith et al.[23] and, Simpson and Palacios [3]. The wing has a $16 \mathrm{~m}$ half span length in spanwise direction with NACA0012 airfoil cross section and subjected to free stream with $U_{\infty}=25 \mathrm{~m} / \mathrm{s}$ and density of $\rho_{\infty}=0.08891 \mathrm{~kg} / \mathrm{m}^{3}$ corresponding to an altitude of $20 \mathrm{~km}$. Hence, the chord-based Reynolds number becomes $R e_{c}=\rho_{\infty} U_{\infty} c / \mu=1.56 \times 10^{5}$. The flow angle of attack (aoa) of $\alpha=4^{\circ}$ and gravitational force of $g=9.754 \mathrm{~m} / \mathrm{s}^{2}$ is considered in current simulations. All parameters are non-dimensionalized with appropriate references. The cord length $c=1 \mathrm{~m}$ is used as a reference length, velocity and time are non-dimensionalized as $\mathbf{u}^{*}=\mathbf{u} / U_{\infty}$ and $t^{*}=t U_{\infty} / c$.

(a)

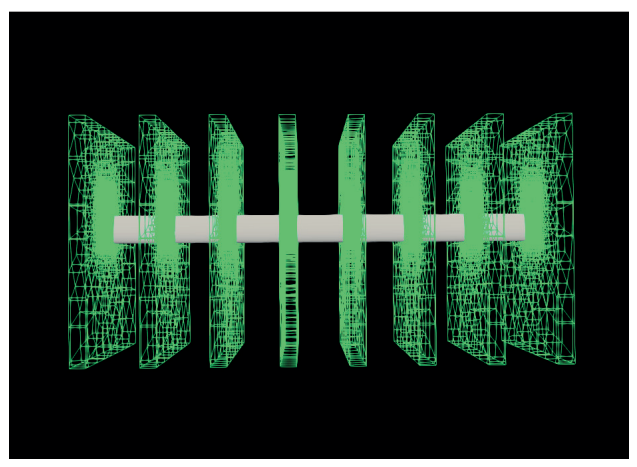

(c)

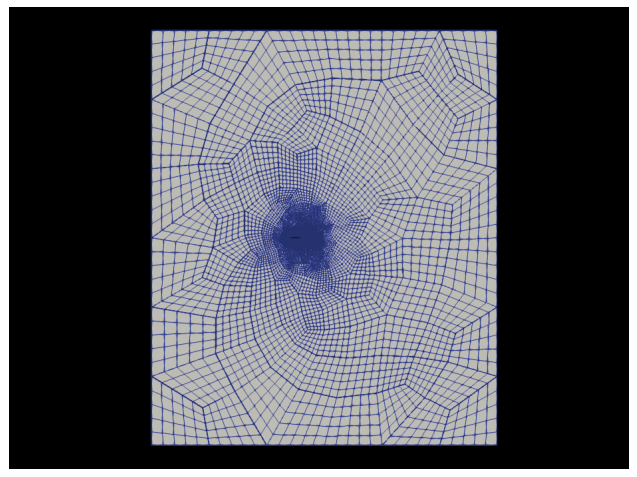

(b)

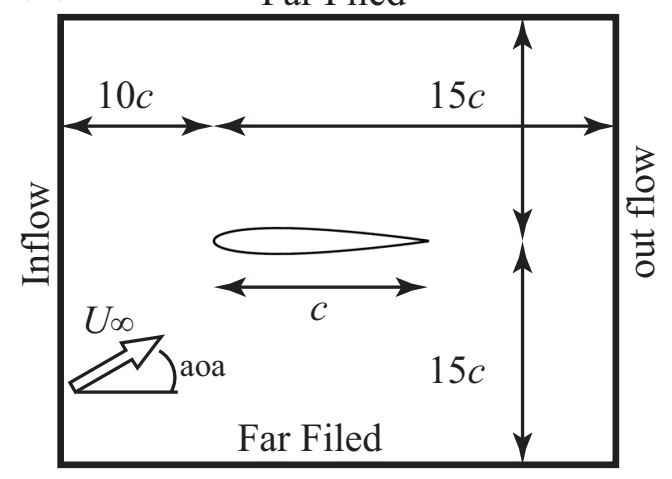

(d)

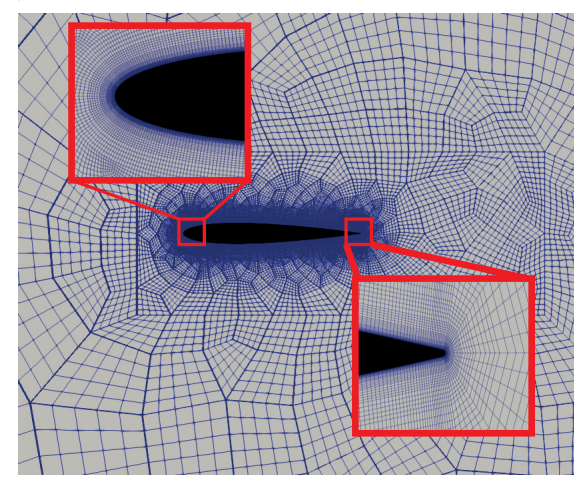

Fig. 2 (a) Distribution of thick strips domains along the HALE wing ( $N=8$ ) (b) Schematic of 2D planes with domain dimensions and boundary conditions (c,d) Computational grid with enlarged view near airfoil leading and trailing edges

The computational domain for the fluid flow consists of 8 fluid strips with the spanwise length of $L_{z}=0.2 c$ as shown in Fig. 2(a). Each strip consists of rectangular $2 D(x, y)$ planes perpendicular to the local axis of structure. The airfoil is placed in the middle of the domain with its leading edge at $(x, y)=(0,0)$. The inlet boundary is placed $10 c$ upstream of the airfoil with the inlet boundary condition specified as $\mathbf{u}^{*}=\left(U_{\infty}^{*} \cos (\alpha), U_{\infty}^{*} \sin (\alpha), 0\right)$ with $U_{\infty}^{*}=1$. The outlet 
Table 1 HALE wing structural properties

\begin{tabular}{ll}
\hline chord $c$ & $1 \mathrm{~m}$ \\
semi-span & $16 \mathrm{~m}$ \\
Elastic axis & $0.5 c$ \\
Center of gravity & $0.5 c$ \\
Mass per unit length & $0.75 \mathrm{~kg} / \mathrm{m}$ \\
Moment of inertia & $0.1 \mathrm{~kg} \cdot \mathrm{m}$ \\
Torsional stiffness & $1 \times 10^{4} \mathrm{Nm}^{2}$ \\
Bending stiffness & $2 \times 10^{4} \mathrm{Nm}^{2}$ \\
Chordwise bending stiffness & $5 \times 10^{6} \mathrm{Nm}^{2}$
\end{tabular}

boundary is located $15 c$ downstream with the high order Neumann boundary condition [1]. Lateral boundaries are located $\pm 15 c$ from the Center of the domain with the free stream boundary condition. Fig. 2(b) shows the schematic of $2 D$ planes including the domain dimension and summarizing the boundary conditions. Further, each $2 D$ planes are discretized with total number of 1704 quadrilateral elements. Boundary layer mesh is used near the airfoil wall with the first layer height of $h_{w}^{*}=4.5 \times 10^{-4}$ and total number of 8 layers which results in maximum $y^{+}=2$ on the suction surface of the foil during the simulations. For all simulations, the polynomial order $P=5$ is used in $(x, y)$ plane for spectral/hp discretization. Fig. 1 (c) depicts the overview of computational grid where an enlarge view near the airfoil, leading and trailing edges shown in Fig. 1 (d).

Due to the explicit treatment of convective terms and high Re number flow, simulations requires small time steps to satisfy the CFL restrictions. Karniadakis and Sherwin [19] showed that the CFL for spectral/hp element discretization can be estimated as

$$
C F L=\max _{\text {elements }} 0.2 \Delta t\left|\mathbf{V}^{s t}\right| P^{2}
$$

where $C F L$ is the Courant-Friedrichs-Lewy condition, $\left|\mathbf{V}^{s t}\right|$ is the maximum velocity in standard element and $P$ is the polynomial order. All simulations, both FSI and stationary airfoil, are performed with fixed time step size of $\Delta t_{f}^{*}=2.5 \times 10^{-5}$ for fluid solver which results in maximum $C F L$ number of 0.4 during the solution.

For the structural solver, 203 -nodes quadratic elements is used to discretized the structure in spanwise direction[3] with 500 load steps to linearly approach final state in each time step. Minimum residual of $\epsilon=1 \times 10^{-8}$ is considered as a convergence criteria in each time step. The time step for the structural dynamics is set to $\Delta t_{s}=\gamma \Delta t_{f}^{*} c / U_{\infty}=1 \times 10^{-3} s$ where $\gamma=1000$ is the frequency at which the aerodynamic forces are updated on the structure and the wing dynamic is evolved one step in time. Ideally, the fluid and structure should be solved in each time step but due to very small fluid time steps, this results in extremely small time steps for the structure and introduces high-frequency excitations into fluid solution domain. Therefore, to alleviate this problem, the structure is evolved one step every $\gamma$ steps of fluid solvers. Further, the aerodynamic forces from the fluid is averaged over the $\gamma$ interval and transferred to the structural solver. Finally, the Structural properties of the wing is summarised in table 1

\section{A. Preliminary result for HALE wing with $\alpha=4^{\circ}$}

In this section preliminary result of the simulation for angle of attack $\alpha=4^{\circ}$ is presented and the three-dimensional effect of spanwise length of strips $L_{z}^{*}$ is discussed for the stationary airfoil. The simulation is performed using 8 fluid strips [Fig. 1 (a)] with two planes (one complex Fourier mode) per strip. Figure 3 (a) shows the the deformation profile of HALE wing at 6 distinct times, starting from $t^{*}=0$ and ending at wing final deformation state at $t^{*}=0.4$ [solid red line in Fig. 3 (a)]. The wing is deformed under its weight at $t^{*}=0$ with the tip deflection of $y_{t i p}^{*}=-3.0177$ and moves gradually upward in $+y$ direction as the result of lift from the flow until $t^{*}=0.4$ where the wing reaches the final static deflection [solid red line in Fig. 3 (a)] with the tip deflection of $y_{t i p}^{*}=1.92$ [Fig. 3(b)]. Further, the wing reaches the steady state static deflection after $t^{*}=0.29$ and the wing tip does not deform significantly after this time [Fig. 3 (b)]. Comparing our simulation result with the one reported in [3] [dashed black line in Fig. 3(a)] shows difference in the final wing deformation between our results and [3]. The wing tip deformation predicted using nonlinear static simulation of the wing with the aerodynamic forces predicted with Unsteady Vortex Lattice Method (ULVM)[3] using SHARPy solver [2], predicts the final position of wing tip at $y_{t i p}^{*}=3.47$ while in our simulation using the dynamic 
(a)

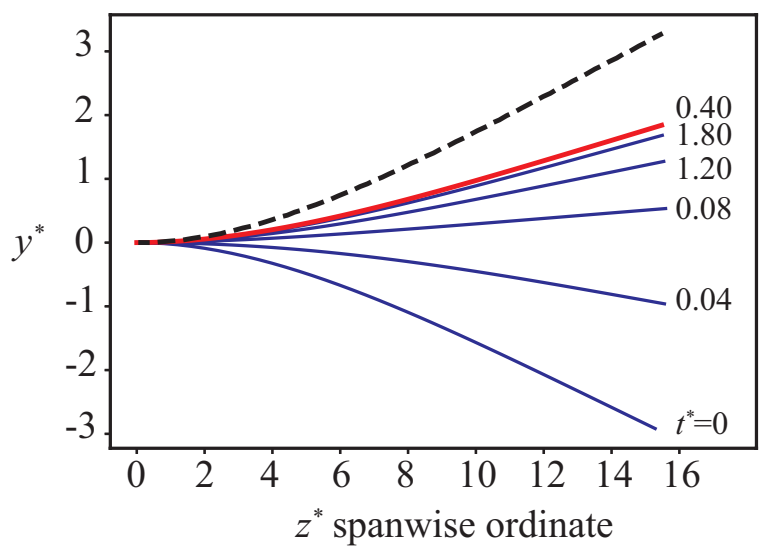

(b)

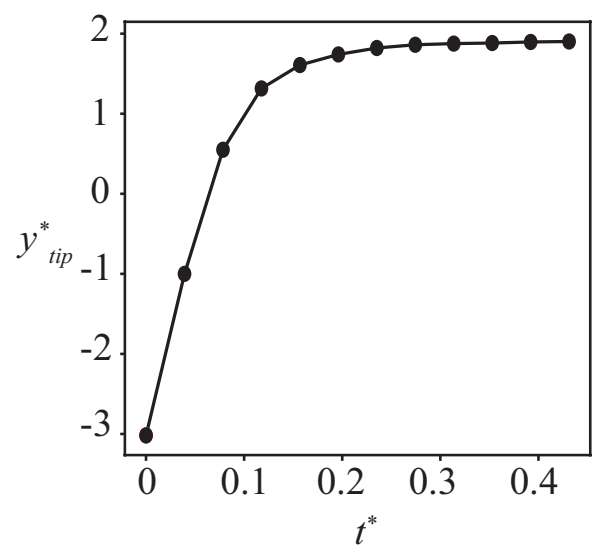

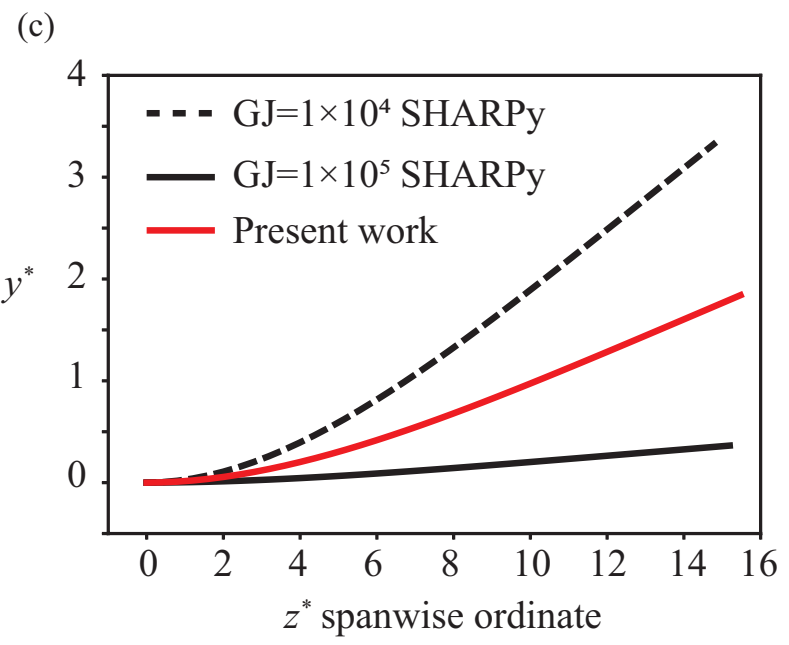

Fig. 3 (a) Time history of HALE wing deformation predicted with current FSI method (solid lines) and the results from [3]. The FSI results obtained using 8 strips, each with one complex Fourier mode (2 planes) in spanwise direction for $R e=1.56 \times 10^{5}$ and angle of attack of $\alpha=4^{\circ}$ (b) Time history of tip deflection from current FSI simulation (c) Effect of torsional stiffness on the wing deformation at its final state, black dashed line SHARPy simulation with $G J=1 \times 10^{4} \mathbf{N m}^{2}$, black solid line $\boldsymbol{S H A R P y}$ simulation with $G J=1 \times 10^{5} \mathrm{Nm}^{2}$ and red line is the result of present simulation.

solution of structural motion coupled with thick strip LES flow solver, the final wing tip is predicted to deform to $y_{\text {tip }}^{*}=1.92$. One reason for this difference, is that currently the torsional effect is not yet implemented in our simulation and the wing does not allow to twist due to aerodynamic moments. Hence, the local angle of attack at wing's sections is only affected by translational movements of the beam which in turn results in lower lift compared to the case with twist. To demonstrate the effect of torsion on the final wing deformation, two tests are simulated using the $S H A R P y$ solver with ULVM method to predict aerodynamic forces and moments. The first case, test A, is with the same setting presented in table 1 with torsional stiffness of $G J=1 \times 10^{4} \mathrm{Nm}^{2}$. The second case, test B, has all structural parameters the same as the test A but the torsional stiffness, which is increased to $G J=1 \times 10^{5} \mathrm{Nm}^{2}$, in order to reduce the angular motion of wing. Fig 3 (c) compares the results of test A, B and the current work. It is clearly evident that the final state of wing deformation decreases with increasing the torsional stiffness [black lines in Fig. 3. (c)]. The wing tip deflection is $y_{t i p}^{*}=3.47$ for the test A which has torsional stiffness of $G J=1 \times 10^{4} \mathrm{Nm}^{2}$ [dashed black line in Fig. 3 (c)]. Increasing the torsional stiffness by factor of $10\left(G J=1 \times 10^{5} \mathrm{Nm}^{2}\right)$ in test $\mathrm{B}$, the tip deflection reduces to $y_{t i p}^{*}=0.39$. Our simulation result for wing tip deflection $\left(y_{t i p}^{*}=1.92\right)$ with thick strip LES approach and torsional 
Table 2 Lift coefficient comparison for various our test cases with the available data in literature

\begin{tabular}{llcccc}
\hline & aoa & $R e$ & $C_{L}$ & $L_{z}^{*}$ & No. of Fourier Planes \\
\hline Present work 2D & $4^{\circ}$ & $1.56 \times 10^{5}$ & 0.422 & 0.1 & 2 \\
Present work Q3D-a & $4^{\circ}$ & $1.56 \times 10^{5}$ & 0.5044 & 0.1 & 8 \\
Present work Q3D-b & $4^{\circ}$ & $1.56 \times 10^{5}$ & 0.5001 & 0.15 & 8 \\
Present work Q3D-c & $4^{\circ}$ & $1.56 \times 10^{5}$ & 0.4769 & 0.2 & 4 \\
Present work Q3D-d & $4^{\circ}$ & $1.56 \times 10^{5}$ & 0.4931 & 0.2 & 8 \\
Present work Q3D-e & $4^{\circ}$ & $1.56 \times 10^{5}$ & 0.5065 & 0.2 & 16 \\
\hline Lehmkuhl et al [24] LES & $5^{\circ}$ & $5.00 \times 10^{4}$ & 0.561 & - & - \\
Lehmkuhl et al [24] DNS & $5^{\circ}$ & $5.00 \times 10^{4}$ & 0.569 & - & - \\
Serson et al [25] DNS & $6^{\circ}$ & $5.00 \times 10^{4}$ & 0.6312 & - & - \\
Paula et al [26] Exp & $4^{\circ}$ & $2.90 \times 10^{5}$ & 0.4902 & - & - \\
Abbot et al [27] Exp & $5^{\circ}$ & $6.00 \times 10^{6}$ & 0.5516 & - & - \\
Ladson [28] Exp & $4^{\circ}$ & $6.00 \times 10^{6}$ & 0.43 & - & - \\
Gregory and O’reilly [29] Exp & $6^{\circ}$ & $6.00 \times 10^{6}$ & 0.6581 & - & -
\end{tabular}

(a)

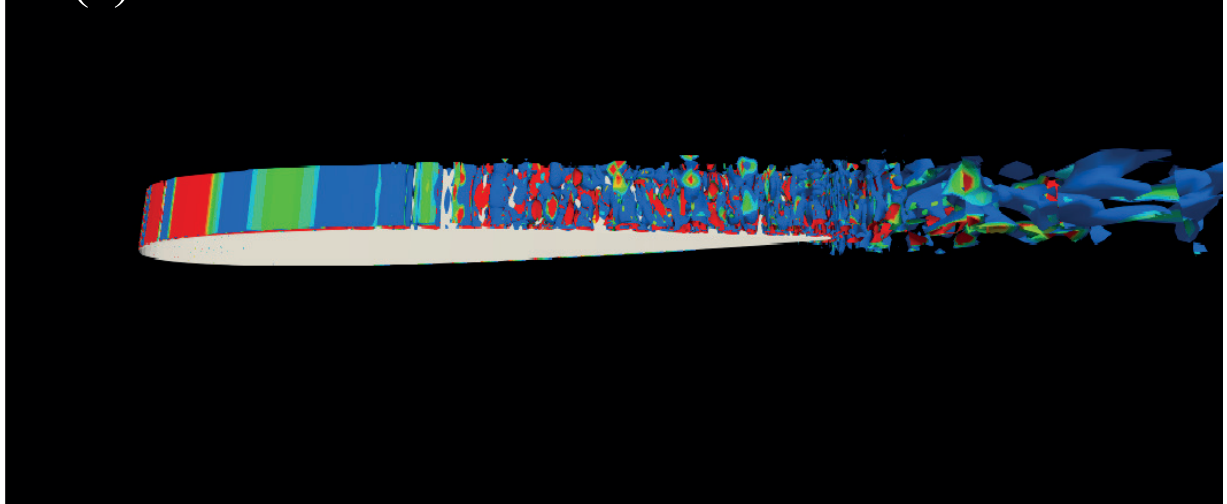

(b)

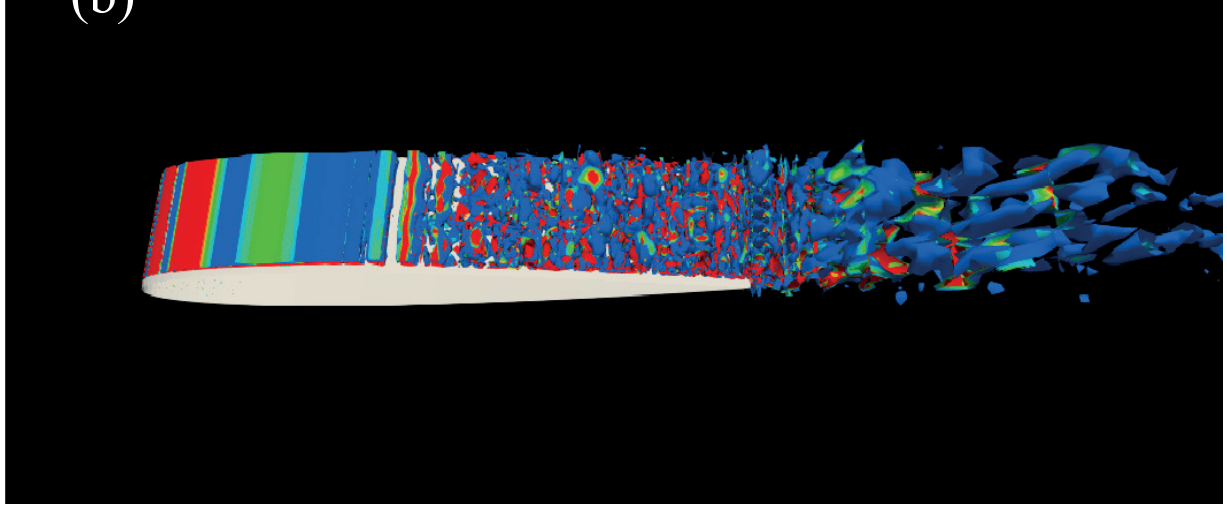

Fig. 4 Contours of Q-criterion at $t^{*}=140$ for (a) Thick strip with $L_{z}^{*}=0.1$ (case Q3D-a in table 2) (b) Thick strip with $L_{z}^{*}=0.2$ (case Q3D-e in table 2). Both cases have the same resolution of 8 Fourier planes in $10 \%$ of chord in spanwise direction. 
stiffness of $G J=1 \times 10^{4} \mathrm{Nm}^{2}$ lies between the results of test A and B [red line in Fig. 3 (c)]. In fact, in our simulation with thick strip method, the torsional stiffness is the same as test A. However, since the wing's angular motion is not considered the wing shows smaller deformations.

Furthermore, we used one complex Fourier mode in spanwise direction in each strips which practically makes the strips $2 D$ domains and unable to capture the local three-dimensional effects. Hence, series of numerical test is conducted to determine the effect of $L_{z}^{*}=L_{z} / c$ and spanwise resolution on the aerodynamic forces. To reduce the computational expenses these numerical tests are performed on a stationary wing with the same grid used for FSI simulation. The first case is the $2 D$ simulation, while the rest of test cases are quasi- $3 D$, homogenous in spanwise direction, with the strip spanwise thickness $L_{z}^{*}=0.1,0.15,0.2$ and two resolution 8 to 16 Fourier planes in spanwise direction. Table 2 summarizes the conducted test cases and compares the $C_{L}$ coefficient with available numerical and experimental data in literature. It should be noted that some of the data from the literature are the digitized reproduction and indicate only approximate values. Further, a comparison between $C_{L}$ data from the literature (table 2 ) indicate that $C_{L}$ is not too sensitive to $R e$ number for the range of $R e$ shown in the table 2 and the lift coefficient is expected to be about $C_{L}=0.5$. However, our $2 D$ test predicts the lift coefficient to be $C_{L}=0.422$ which is about $15 \%$ lower than that predicted with quasi-3D simulations (table 2). Further, comparing the results for the cases Q3D-c, Q3D-d and Q3D-e which all have same thickness for the fluid domain $\left(L_{z}^{*}=0.2\right)$ but having different resolution in spanwise direction shows that using 4 planes in spanwise direction (Q-3D-c) results in 6\% under-prediction of the lift coefficient compared to the case Q3 $D$-e which has 16 planes in spanwise direction (table 2). Moreover, comparison between cases Q3D-a and Q3D-e which both have same resolution in spanwise direction per unit length, i.e. 8 planes per $10 \%$ of chord length indicates that the results are not sensitive to the spanwise length of the domain as the $C_{L}$ in these cases differs less than one percent. Figs. 4 (a) and 4 b) compares the snapshot of Q-criterion at $t^{*}=140$ for Q-3D-a and Q-3D-e which shows similar turbulent structure for these two cases and further corroborates that as long as the simulation have adequate spanwise resolution, the results are independent of $L_{z}^{*}$ for $L_{z}^{*} \geq 0.1$. Therefore, based on the results of the $2 D$ and Q3D-a to Q3D-e tests for stationary NACA0012 foil, it can be inferred that lack of resolution in spanwise direction of thick strips is also contributes to a smaller deformation of HALE wing.

To conclude this section, we summarize that an efficient computational method is present for high fidelity simulation of flexible wings in stall. Large eddy simulation with thick strip method is used to efficiently break down the problem into smaller $3 D$ fluid domains and structural dynamics is solved using geometrically-exact composite beam for highly deformable slender structures. Preliminary results shows capability and robustness of presented approach for simulation of flexible wings in stall. Higher resolution in spanwise direction and angular motion are being developed and are planned for future work of this paper.

\section{Future work}

Following the preliminary results presented, the paper aims to further extends the simulations to incorporate the $3 D$ effects in thick strips. Study of the effect of number of thick strips as well as HALE wing deformation at various angle of attacks and static stall prediction are future planes of this paper. In addition to this, for prediction of static stall hysteresis curve, restarting the solution from the previous structural dynamic state should be incorporated in the couple

FSI solver. Finally the torsional movement of the wing due to aerodynamic moments, which is currently neglected, needs to be considered in the FSI solver in order to achieve more accurate simulations. These steps will be addressed in the final version of this work.

\section{Acknowledgments}

This work is supported by European Commission (EC) under H2020-EU.1.2.2.-FET Proactive program and the Research and Innovation action (RIA) funding scheme, through the project High Performance Computing in Wind Energy (HPCWE) (grant agreement ID. 828799)

\section{References}

[1] Bao, Y., Palacios, R., Graham, M., and Sherwin, S., "Generalized thick strip modelling for vortex-induced vibration of long flexible cylinders," Journal of Computational Physics, Vol. 321, 2016, pp. 1079-1097.

[2] Muñoz-Simón, A., Wynn, A., and Palacios, R., "Unsteady and three-dimensional aerodynamic effects on wind turbine rotor loads," AIAA Scitech 2020 Forum, 2020, p. 0991. 
[3] Simpson, R. J., and Palacios, R., "Numerical aspects of nonlinear flexible aircraft flight dynamics modeling," 54th AIAA/ASME/ASCE/AHS/ASC Structures, Structural Dynamics, and Materials Conference, 2013, p. 1634.

[4] Chaplin, J., Bearman, P., Cheng, Y., Fontaine, E., Graham, J., Herfjord, K., Huarte, F. H., Isherwood, M., Lambrakos, K., Larsen, C., et al., "Blind predictions of laboratory measurements of vortex-induced vibrations of a tension riser," Journal of fluids and structures, Vol. 21, No. 1, 2005, pp. 25-40.

[5] Facchinetti, M. L., De Langre, E., and Biolley, F., "Coupling of structure and wake oscillators in vortex-induced vibrations," Journal of Fluids and structures, Vol. 19, No. 2, 2004, pp. 123-140.

[6] Gabbai, R., and Benaroya, H., "An overview of modeling and experiments of vortex-induced vibration of circular cylinders," Journal of Sound and Vibration, Vol. 282, No. 3-5, 2005, pp. 575-616.

[7] Hau, E., Wind turbines: fundamentals, technologies, application, economics, Springer Science \& Business Media, 2013.

[8] Wang, L., Liu, X., Renevier, N., Stables, M., and Hall, G. M., "Nonlinear aeroelastic modelling for wind turbine blades based on blade element momentum theory and geometrically exact beam theory," Energy, Vol. 76, 2014, pp. 487-501.

[9] Shearer, C. M., and Cesnik, C. E., "Nonlinear flight dynamics of very flexible aircraft," Journal of Aircraft, Vol. 44, No. 5, 2007, pp. 1528-1545.

[10] Peters, D. A., "Two-dimensional incompressible unsteady airfoil theory-an overview," Journal of Fluids and Structures, Vol. 24, No. 3, 2008, pp. 295-312.

[11] Palacios, R., Murua, J., and Cook, R., "Structural and aerodynamic models in nonlinear flight dynamics of very flexible aircraft," AIAA journal, Vol. 48, No. 11, 2010, pp. 2648-2659.

[12] Katz, J., and Plotkin, A., Low-speed aerodynamics, Vol. 13, Cambridge university press, 2001.

[13] Cantwell, C. D., Moxey, D., Comerford, A., Bolis, A., Rocco, G., Mengaldo, G., De Grazia, D., Yakovlev, S., Lombard, J.-E., Ekelschot, D., et al., "Nektar++: An open-source spectral/hp element framework," Computer physics communications, Vol. 192, 2015, pp. 205-219.

[14] del Carre, A., Muñoz-Simón, A., Goizueta, N., and Palacios, R., "SHARPy: A dynamic aeroelastic simulation toolbox for very flexible aircraft and wind turbines," Journal of Open Source Software, Vol. 4, No. 44, 2019, p. 1885.

[15] Simpson, R. J., "Unsteady aerodynamics, reduced-order modelling, and predictive control in linear and nonlinear aeroelasticity with arbitrary kinematics,” Ph.D. thesis, Imperial College London, 2015.

[16] Newman, D., and Karniadakis, G., "Simulations of flow over a flexible cable: a comparison of forced and flow-induced vibration," Journal of fluids and Structures, Vol. 10, No. 5, 1996, pp. 439-453.

[17] Li, L., Sherwin, S., and Bearman, P. W., "A moving frame of reference algorithm for fluid/structure interaction of rotating and translating bodies," International Journal for Numerical Methods in Fluids, Vol. 38, No. 2, 2002, pp. 187-206.

[18] Karniadakis, G. E., Israeli, M., and Orszag, S. A., "High-order splitting methods for the incompressible Navier-Stokes equations," Journal of computational physics, Vol. 97, No. 2, 1991, pp. 414-443.

[19] Karniadakis, G., and Sherwin, S., Spectral/hp element methods for computational fluid dynamics, Oxford University Press, 2013.

[20] Hesse, H., "Consistent aeroelastic linearisation and reduced-order modelling in the dynamics of manoeuvring flexible aircraft," Ph.D. thesis, Imperial College London, 2013.

[21] Del Carre, A., and Palacios, R., "Low-altitude dynamics of very flexible aircraft," Proceedings of the AIAA Scitech 2019 Conference, San Diego, California, USA. AIAA Paper, 2019.

[22] Patil, M. J., and Hodges, D. H., "On the importance of aerodynamic and structural geometrical nonlinearities in aeroelastic behavior of high-aspect-ratio wings," Journal of Fluids and Structures, Vol. 19, No. 7, 2004, pp. 905-915.

[23] Smith, M., Patil, M., and Hodges, D., "CFD-based analysis of nonlinear aeroelastic behavior of high-aspect ratio wings," 19th AIAA Applied Aerodynamics Conference, 2001, p. 1582.

[24] Lehmkuhl, O., Rodríguez, I., Baez, A., Oliva, A., and Pérez-Segarra, C., "On the large-eddy simulations for the flow around aerodynamic profiles using unstructured grids," Computers \& Fluids, Vol. 84, 2013, pp. 176-189. 
[25] Serson, D., Meneghini, J. R., and Sherwin, S. J., "Direct numerical simulations of the flow around wings with spanwise waviness," Journal of Fluid Mechanics, Vol. 826, 2017, pp. 714-731.

[26] Paula, A. A. d., "The airfoil thickness effects on wavy leading edge phenomena at low Reynolds number regime.” Ph.D. thesis, Universidade de São Paulo, 2016.

[27] Abbott, I. H., and Von Doenhoff, A. E., Theory of wing sections: including a summary of airfoil data, Courier Corporation, 2012.

[28] Ladson, C. L., Effects of independent variation of Mach and Reynolds numbers on the low-speed aerodynamic characteristics of the NACA 0012 airfoil section, Vol. 4074, National Aeronautics and Space Administration, Scientific and Technical ..., 1988.

[29] Gregory, N., and O'reilly, C., "Low-speed aerodynamic characteristics of NACA 0012 aerofoil section, including the effects of upper-surface roughness simulating hoar frost," 1970. 